\title{
Martin Luther King, 40 ans après sa disparition
}

Entre histoire et mémoire

\section{Hélène Harter}

\section{OpenEdition}

Journals

Édition électronique

URL : http://journals.openedition.org/echogeo/10683

DOI : $10.4000 /$ echogeo.10683

ISSN : 1963-1197

Éditeur

Pôle de recherche pour l'organisation et la diffusion de l'information géographique (CNRS UMR 8586)

Référence électronique

Hélène Harter, « Martin Luther King, 40 ans après sa disparition », EchoGéo [En ligne], Sur le Vif, mis en ligne le 14 janvier 2009, consulté le 30 avril 2019. URL : http://journals.openedition.org/ echogeo/10683; DOI : 10.4000/echogeo.10683

Ce document a été généré automatiquement le 30 avril 2019

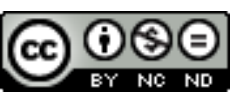

EchoGéo est mis à disposition selon les termes de la licence Creative Commons Attribution - Pas d'Utilisation Commerciale - Pas de Modification 4.0 International 


\title{
Martin Luther King, 40 ans après sa disparition
}

\author{
Entre histoire et mémoire
}

\section{Hélène Harter}

1 Le 4 avril 1968, les Américains apprennent avec stupéfaction que Martin Luther King vient d'être assassiné. Alors qu'il discutait avec quelques proches sur le balcon du motel Lorraine dans le quartier noir de Memphis (Tennessee), il a été abattu par un tireur embusqué. Il n'avait que 39 ans. L'émotion est immense aux Etats-Unis, mais également dans le reste du monde. Les Américains pleurent l'homme qui incarne la lutte pour les droits civiques. Ils sont bouleversés par cet assassinat politique qui intervient moins de cinq ans après la mort du président Kennedy. Quarante après, le souvenir de M. L. King demeure bien vivant. En cette année 2008, son combat prend même une nouvelle résonnance alors que les Américains élisent pour la première fois de leur histoire un président issu de la communauté noire. Cet anniversaire nous donnera l'occasion de retracer le parcours de M.L. King, de revenir sur les événements qui ont suivi sa mort mais également de comprendre comment sa mémoire s'est construite aux États-Unis.

\section{De la lutte pour les droits civiques au combat pour l'égalité économique}

2 En à peine quelques années, Martin Luther King Jr est devenu le leader du combat des Noirs américains pour les droits civiques. C'est en 1955 qu'il est devenu une figure nationale. Jeune pasteur à Montgomery (Alabama), il s'investit dans la campagne de boycottage des bus organisée par les associations locales de lutte pour les droits civiques. M. L. King a bien compris qu'à l'heure de la télévision il fallait mener des opérations spectaculaires pour attirer l'attention des médias et de l'opinion et faire évoluer les choses. Homme d'église - les églises jouent un rôle de premier plan dans la société américaine, et en particulier dans la communauté noire ${ }^{1}$-, orateur inspiré - il suffit de songer à son discours I Have a Dream prononcé le 28 août 1963 pour le $100^{\mathrm{e}}$ anniversaire de l'abolition de l'esclavage -, organisateur de talent, homme de courage - son militantisme 
le conduit 29 fois en prison -, il choisit de recourir à la désobéissance civile et à la nonviolence pour faire progresser la condition des Noirs américains.

L'arrêt Brown de la Cour suprême a rendu la ségrégation illégale dans les écoles primaires publiques en 1954. Les lois sur les droits civiques de juillet 1964 et août 1965 ont donné la pleine égalité civique à tous les Américains. Mais dans le Sud la déségrégation est lente. A Memphis, les éboueurs, en majorité noire, sont en grève depuis le début de l'année 1968 pour réclamer de meilleures conditions de travail et surtout la fin des discriminations raciales. En vain. C'est pourquoi les organisations de défense des droits civiques de la ville demandent au révérend King de leur apporter son aide ${ }^{2}$. Elles espèrent que sa notoriété donnera une résonance nationale à leur combat et leur permettra d'obtenir satisfaction. Martin Luther King accepte. Deux fois, il se rend à Memphis. Mais sa présence ne brise pas la détermination des autorités blanches qui gouvernent la ville. Il accomplit alors un troisième déplacement le 3 avril. Ce soir là, lors d'un grand discours devant 20000 personnes, il annonce son intention de manifester en dépit de l'interdiction des tribunaux. Il explique : «[...] Nous sommes déterminés à obtenir notre juste place dans ce monde du Bon Dieu. Et c'est là, tout ce dont il s'agit. Nous ne sommes engagés dans une aucune protestation négative, dans aucune discussion négative vis-à-vis de personne. Nous disons que nous sommes déterminés à être des hommes. Nous sommes déterminés à être des personnes. Nous affirmons, nous affirmons que nous sommes des enfants du Bon Dieu. Et si nous sommes des enfants du Bon Dieu, nous n'avons pas à vivre comme on veut nous forcer à vivre $»^{3}$. L'égalité civique a été obtenue en 1964 et 1965 . Désormais, le révérend King fait de la lutte contre la pauvreté son cheval de bataille. Celle-ci touche $60 \%$ des Noirs. C'est trois fois plus que les Blancs. La manifestation prévue à Memphis le 5 avril doit d'ailleurs être une répétition grandeur nature avant la grande marche des pauvres prévue quelques jours plus tard à Washington.

4 Martin Luther King est une figure respectée des militants des droits civiques de Memphis mais en ce printemps 1968 il est loin de faire l'unanimité aux États-Unis ${ }^{4}$. A l'hostilité traditionnelle des Blancs du Sud, vient s'ajouter celle de certains de ses soutiens démocrates traditionnels qui ne lui pardonnent pas ses prises de positions contre la guerre du Vietnam. La lenteur des réformes est source d'impatience chez les Noirs de la jeune génération qui ne se reconnaissent pas dans la lutte non-violente. En 1965 et 1967, les ghettos sont d'ailleurs secoués par des émeutes. En outre, alors que le révérend King milite pour que les Noirs soient des membres à part entière de la communauté nationale, le Black Power de Stokely Carmichael glorifie une identité noire séparée et fait de plus en plus d'émules. La manifestation qui a lieu le 28 mars 1968 à Memphis témoigne bien de cette évolution. Infiltrée par de jeunes extrémistes noirs, elle finit dans la violence malgré la présence de Martin Luther King.

\section{Une mort à l'origine de nouvelles violences}

5 Le révérend King est un homme en perte d'influence au printemps 1968 mais son assassinat en fait un martyr et un mythe. A l'annonce de sa disparition, les ghettos d'une centaine de villes s'embrasent. Des troubles éclatent à Chicago et Baltimore. Même la capitale fédérale, Washington, n'est pas épargnée. Voulant venger sa mort, de jeunes Noirs descendent dans les rues de leur quartier et pillent les commerces tenus par les Blancs. Les émeutes sont spontanées mais elles sont rapidement entretenues par les mouvements noirs extrémistes qui en appellent à la lutte « contre l'Amérique blanche ». 
L'émotion dépasse cependant la communauté noire. Le président Johnson déclare un deuil national. Des obsèques publiques sont organisées à Atlanta, la ville natale du révérend. Cent mille personnes s'y pressent dont de nombreuses personnalités blanches comme le vice-président Humphrey et la veuve de John Kennedy. Ils sont cent fois plus nombreux à suivre l'événement devant leur télévision. Cinq ans après l'assassinat du président Kennedy, alors que les Etats-Unis sont engagés dans une campagne présidentielle très disputée sous fond de contestation dans les campus et de manifestations anti-guerre, les Américains s'inquiètent de ce nouvel acte de violence politique $^{5}$. Les rumeurs de complot s'intensifient alors qu'on ne sait toujours pas qui a tiré sur M. L. King.

6 Grâce à une vaste enquête du FBI, son assassin est finalement arrêté le 8 juin, à l'aéroport d'Heathrow en Angleterre. C'est un sudiste blanc de 40 ans qui s'appelle James Earl Ray. Echappé du pénitencier du Missouri, il a un lourd passé de délinquant. Au-delà du racisme, ses motivations sont obscures. On parle d'un contrat qu'auraient placé sur la tête du révérend King deux avocats de Memphis, John Sutherland et John Kauffmann ${ }^{6}$. Les hypothèses l'emportent sur les certitudes. J. E. Ray ayant plaidé coupable, il n'y a pas de procès. Les choses se compliquent lorsque, condamné à 99 ans de prison, il commence à clamer son innocence ; une ligne de conduite qu'il conserve jusqu'à sa mort en avril 1998. Les rumeurs de complot enflent à un tel point que la chambre des Représentants décide en 1978 de créer une commission d'enquêtes sur les assassinats du président Kennedy et de Martin Luther King. Ses conclusions sont rendues publiques en mars 1979. Elles innocentent les autorités locales et fédérales de toute complicité et renforcent l'hypothèse Sutherland; sans toutefois pleinement convaincre les Américains.

\section{La mémoire de Martin Luther King}

7 Le combat des partisans du pasteur King ne s'arrête pas avec sa mort. Dans son dernier discours à Memphis, ne disait-il pas: "Comme tout le monde, je voudrais vivre longtemps. La longévité a son prix. Mais je ne m'en soucie guère maintenant. Je veux simplement que la volonté de Dieu soit faite. Et il m'a permis d'atteindre le sommet de la montagne. J'ai regardé autour de moi. Et j'ai vu la Terre promise. Il se peut que je n'y pénètre pas avec vous. Mais je veux vous faire savoir, ce soir, que notre peuple atteindra la Terre promise. Ainsi, je suis heureux, ce soir. Je ne m'inquiète de rien. Je ne crains aucun homme. Mes yeux ont vu la gloire de la venue du Seigneur ${ }^{7}$. Ses amis reprennent le flambeau : ses compagnons de la première heure, comme Ralph Abernathy, avec qui il a fondé la Southern Christian Leadership Conference (conférence des leaders chrétiens du Sud) en 1957, mais également de jeunes militants comme Andrew Young ${ }^{8}$ et Jesse Jackson. Ce dernier fait ainsi campagne pour l'investiture démocrate lors des élections présidentielles de 1984 et 1988. Il échoue. Sa candidature ne dépasse pas la communauté noire et les progressistes blancs. Il est vrai que la situation des Noirs ne constitue plus une priorité politique depuis les années 1970. A force de lobbying, les proches de Martin Luther King obtiennent néanmoins du Congrès qu'une journée du calendrier civique américain lui soit consacrée au moment de l'anniversaire de sa naissance. C'est ainsi que depuis 1986 les Américains célèbrent officiellement sa mémoire le troisième lundi de janvier ${ }^{9}$. Grâce au Martin Luther King Day, les jeunes générations nées bien après sa disparition découvrent son œuvre. 
8 Tout comme cet anniversaire, celui de sa disparition est le prétexte à de nombreux articles dans les médias. C'est encore plus manifeste lors des grandes dates comme le $40^{\mathrm{e}}$ anniversaire en 2008. En cette année électorale, les candidats à la présidentielle ne manquent pas de rappeler l'héritage du pasteur. Ces propos prennent d'autant plus de sens que l'un des principaux candidats, Barack Obama, est un métis dont le père est noir. Le 4 avril, depuis l'Indiana où il fait campagne, il prononce un discours lequel il rappelle que «le docteur King avait compris que la lutte pour la justice économique et pour la justice raciale ne faisaient qu'une, que chacune faisait partie d'une lutte plus grande pour la liberté, la dignité et l'humanité $»^{10}$. Hillary Clinton, qui a été une jeune militante des droits civiques dans les années 1960, et John McCain, un des candidats républicains, se rendent à Memphis pour l'occasion. Ces anniversaires donnent également aux chercheurs et aux journalistes l'occasion d'évaluer dans quelle mesure la condition des Noirs a changé depuis les années 1960. Un sondage Gallup réalisé à l'occasion du Martin Luther King Day de 2008 montre ainsi que $43 \%$ des Américains pensent que les buts de Martin Luther King ont été atteints ; un chiffre faible, mais en net progrès par rapport à 1997, où seuls $26 \%$ des Américains partageaient cette opinion ${ }^{11}$. Plus qu'un sondage, l'élection de Barack Obama à la tête du pays en novembre 2008 témoigne du chemin qu'ont parcouru les Etats-Unis en quatre décennies. Les Noirs ont un taux de pauvreté double de la moyenne nationale. Un quart d'entre eux connaissent encore la précarité et réussissent moins bien que les immigrants. Mais cela ne doit pas faire oublier la réussite du reste de la communauté. Grâce au militantisme des acteurs du mouvement pour les droits civiques, Martin Luther King en tête, grâce à la politique volontariste de l'Administration Johnson qui a fait voter des lois sur les droits civiques et a permis aux Noirs d'accéder en nombre à des emplois publics et à l'enseignement supérieur, la situation des Noirs s'est considérablement améliorée.

9 La lutte pour l'égalité civique n'est plus à l'ordre du jour aux États-Unis. Barack Obama, lui-même, appartient à une génération qui a grandi dans une Amérique déségréguée et multiculturaliste. La question raciale a d'ailleurs été peu présente durant la campagne ; une campagne ${ }^{12}$ durant laquelle $\mathrm{B}$. Obama a réussi à transcender les barrières raciales à la différence de Jesse Jackson dans les années 1980. Il l'a pu d'autant plus qu'il est un métis et que la famille de son père est kenyane et n'a pas connu l'expérience de l'esclavage à la différence de celle de Martin Luther King et des leaders des mouvements des droits civiques. N'oublions pas en outre que depuis le recensement de l'an 2000, ce sont les Hispaniques, et non plus les Noirs, qui sont la première minorité du pays. Bien que la question noire a perdu de son acuité aux Etats-Unis, Martin Luther King n'en demeure pas moins une référence pour les Américains. Un sondage Gallup de décembre 1999 en fait la personnalité du XXe siècle la plus admirée de deux tiers d'entre eux ${ }^{13}$ tandis que de très nombreux livres lui sont encore consacrés: études universitaires mais également témoignages de proches, comme sa sœur Christine King Farris, et ouvrages grands publics ${ }^{14}$. Le $40^{\mathrm{e}}$ anniversaire de sa mort conduit à une multiplication des publications. Mais il n'explique pas à lui seul l'intérêt que les Américains continuent de porter à M. L. King. Il faut surtout y voir la forte résonance qu'a son combat dans un pays qui non seulement s'est construit autour des idéaux de liberté et d'égalité mais demeure également très inégalitaire malgré les progrès accomplis ces quarante dernières années. 


\section{NOTES}

1. Houck, D. W., Dixon, D. E. dir., Rhetoric, Religion and the Civil Rights Movement, 1954-1965, Waco (Texas), Baylor University Press, 2006.

2. Kaspi, A, 1988, Etats-Unis 68 : L'année des contestations, Bruxelles, Complexe, p. 46.

3. Carson, C, 2000, Martin Luther King, Autobiographie, Paris, Bayard, p. 430.

4. D'après un sondage Gallup d'août 1966, seuls 33\% des Américains ont une opinion favorable de M.L.King; un chiffre en net recul par rapport aux années 1963-1964 qui marquent l'apothéose de sa popularité. C'est d'ailleurs en 1964 qu'il obtient le prix Nobel de la paix. http:// www.gallup.com/poll/103828/Civil-Rights-Progress-Seen-More.aspx [consulté le 20 décembre 2008].

5. Les Etats-Unis semblent s'enfoncer encore un peu plus dans la violence avec l'assassinat de Robert Kennedy lors des primaires démocrates de Californie le 5 juin 1968.

6. Kaspi, A, op. cit., p. 59.

7. Carson, C, 2000, op.cit., p. 434-435

8. Andrew Young deviendra en 1977 le premier représentant des Etats-Unis à l'ONU qui soit issu de la communauté noire.

9. Seuls George Washington et Abraham Lincoln (Presidents' Day, le troisième lundi de février) et Christophe Colomb (Columbus Day, le deuxième lundi d'octobre) bénéficient de l'honneur d'une telle journée.

10. http://www.barackobama.com/2008/04/04/remarks_for_senator_barack_oba_4.php. Site officiel de campagne de Barack Obama [consulté le 20 décembre 2008].

11. http://www.gallup.com/poll/103828/Civil-Rights-Progress-Seen-More.aspx [consulté le 20 décembre 2008]

12. Cf. Ghorra-Gobin, $\mathrm{C}$, « La 44 e élection présidentielle :un événement révélateur des mutations de la société américaine ", Echogéo, 8 otobre 2008 (http://echogeo.revues.org/index7363.html; consulté le 9 janvier 2009) et Ghorra-Gobin, C, « La 44 $\underline{\text { élection présidentielle : un événement }}$ révélateur des mutations de la société américaine $: \underline{\text { Le regard des Américains dans un contexte }}$ mondial difficile, Echogéo, 19 novembre 2008 (http://echogeo.revues.org/index9113.html; consulté le 9 janvier 2009).

13. http://www.gallup.com/poll/103828/Civil-Rights-Progress-Seen-More.aspx [consulté le 20 décembre 2008].

14. Parmi les ouvrages scientifiques récents, citons : Jackson, T. F., From Civil Rights to Human Rights : Martin Luther King, Jr., and the Struggle for Economic Justice, Philadelphie, University of Pennsylvania Press, 2007 ; Jackson, T., Becoming King : Martin Luther King, Jr. and the Making of a National Leader, Lexington, University Press of Kentucky, 2008. Les Mémoires de la sœur de M. L. King sont parus sous le titre : King Farris, C., Through it All : Reflections on My Life, My Family, and My Faith, New York, Atria Books, 2009. La littérature pour enfant s'intéresse également à Martin Luther King. Cf : Walker, I., The Assassination of Dr. Martin Luther King Jr., Edina (Minnesota), Abdo Publishing Company, 2008. 


\section{RÉSUMÉS}

Le 4 avril 1968 Martin Luther King est assassiné à Memphis. Moins de cinq ans après la mort du président Kennedy, les Etats-Unis sont à nouveau touchés par la violence politique. Cet article retrace le parcours qui a conduit le révérend King jusqu'à Memphis en cette année politiquement et socialement agitée. Comment sa mort est-elle vécue aux États-Unis? Quelle mémoire conserve-t-on de lui aujourd'hui?

On April 4, 1968 Martin Luther King was assassinated in Memphis. Less than five years after the death of President Kennedy, the United States were affected again by political violence. This article explains why Rev. King went to Memphis during this politically and socially troubled year. How his death has been perceived in the United States? Does his legacy still live on forty years after his death?

\section{INDEX}

Mots-clés : Etats-Unis, histoire, histoire politique, Martin Luther King, noirs américains Keywords : United States, history, political history, Martin Luther King, African-Americans

\section{AUTEUR}

\section{HÉLÈNE HARTER}

Harter, Hélène, helene.harter@univ-paris1.fr, Maître de conférences HDR en histoire contemporaine, Université Paris 1 Panthéon-Sorbonne, Directeur-adjoint du Centre de recherches d'histoire nord-américaine, Hélène Harter, L'Amérique en guerre : les villes pendant la Seconde Guerre mondiale, Paris, Galaade Éditions, 2006 ; en collaboration avec André Kaspi, François Durpaire et Adrien Lherm, La civilisation américaine, Paris, Presses Universitaires de France, Collection Quadrige, 2006 ; Hélène Harter, Antoine Marès, Pierre Melandri et Catherine Nicault dir., Terres promises : Mélanges offerts à André Kaspi, Paris, Publications de la Sorbonne, 2008. 